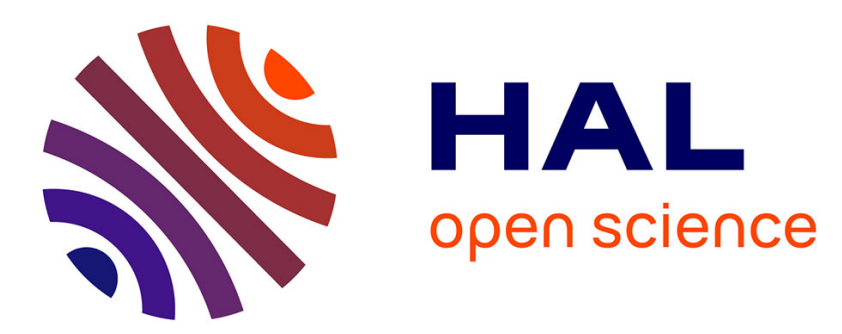

\title{
Comparative assessment of continuum-scale models of bimolecular reactive transport in porous media under pre-asymptotic conditions
}

\author{
G.M. Porta, G. Ceriotti, J.-F. Thovert
}

\section{- To cite this version:}

G.M. Porta, G. Ceriotti, J.-F. Thovert. Comparative assessment of continuum-scale models of bimolecular reactive transport in porous media under pre-asymptotic conditions. Journal of Contaminant Hydrology, 2016, 185-186, pp.1-13. 10.1016/j.jconhyd.2015.12.003 . hal-03124336

\author{
HAL Id: hal-03124336 \\ https://hal.science/hal-03124336
}

Submitted on 28 Jan 2021

HAL is a multi-disciplinary open access archive for the deposit and dissemination of scientific research documents, whether they are published or not. The documents may come from teaching and research institutions in France or abroad, or from public or private research centers.
L'archive ouverte pluridisciplinaire HAL, est destinée au dépôt et à la diffusion de documents scientifiques de niveau recherche, publiés ou non, émanant des établissements d'enseignement et de recherche français ou étrangers, des laboratoires publics ou privés. 
1 Comparative assessment of continuum-scale models of bimolecular reactive transport in porous media under pre-asymptotic conditions

3

by G.M. Porta ${ }^{1}$, G. Ceriotti ${ }^{1}$ and J.-F. Thovert ${ }^{2}$

$8 \quad{ }^{1}$ Dipartimento di Ingegneria Civile ed Ambientale, Politecnico di Milano, Piazza L. Da Vinci 32, 20133 Milano, Italy 


\section{ABSTRACT}

18 We compare the ability of various continuum-scale models to reproduce the key features of a

19 transport setting associated with a bimolecular reaction taking place in the fluid phase and

20 numerically simulated at the pore-scale level in a disordered porous medium. We start by

21 considering a continuum-scale formulation which results from formal upscaling of this reactive

22 transport process by means of volume averaging. The resulting (upscaled) continuum-scale

23 system of equations includes nonlocal integro-differential terms and the effective parameters

24 embedded in the model are quantified directly through computed pore-scale fluid velocity and

25 pore space geometry attributes. The results obtained through this predictive model formulation

26 are then compared against those provided by available effective continuum models which require

27 calibration through parameter estimation. Our analysis considers two models recently proposed

28 in the literature which are designed to embed incomplete mixing arising from the presence of fast

29 reactions under advection dominated transport conditions. We show that best estimates of the

30 parameters of these two models heavily depend on the type of data employed for model

31 calibration. Our upscaled nonlocal formulation enables us to reproduce most of the critical

32 features observed through pore-scale simulation without any model calibration. As such, our

33 results clearly show that embedding into a continuum-scale model the information content

34 associated with pore-scale geometrical features and fluid velocity yields improved interpretation

35 of typically available continuum-scale transport observations.

36 Keywords: reactive transport; continuum-scale models; upscaling; parameter estimation; pore-

37 scale modeling 


\section{INTRODUCTION}

40 Recent developments in pore-scale numerical modeling and imaging technologies are

41 providing remarkable added value to our knowledge of dynamics of reactive transport processes

42 in complex pore spaces (e.g., Molins et al., 2014; Menke et al., 2015). These techniques and

43 approaches allow characterizing and comparing the relative importance of the dynamics

44 associated with advective transport, diffusive mixing and reactive processes at the (micron) scale

45 of individual pores. Reactive transport applications in hydrogeology typically involve large

46 spatial scales. It would then be desirable to explore the extent at which our knowledge on pore-

47 scale dynamics could be transferred into models that can be applied at a continuum- (or Darcy-)

48 scale. In this work we consider solute transport in porous media in the presence of a

49 homogeneous bimolecular irreversible reaction of the kind $\mathrm{A}+\mathrm{B} \rightarrow \mathrm{C}$, which is typically

50 classified as a fluid-fluid reaction. Upscaling this reactive transport problem from the pore- to a

51 continuum-scale is especially challenging under conditions of advection dominated transport and

52 fast reaction, respectively associated with large values of the Péclet, $P e$, and Damköhler, $D a$,

53 numbers (e.g. Gramling et al., 2002; Tartakovsky et al., 2009; Porta et al., 2012a). Available

54 column experiments at the laboratory scale document the space-time dynamics of the reaction

55 product C (Gramling et al., 2002; Raje and Kapoor, 2000). These works show that the

56 assumption of complete mixing of the reactants at the pore-scale can lead to considerable

57 discrepancies between measured concentration of the reaction product $\mathrm{C}$ and predictions based

58 on continuum-scale approximations. This result is consistent with theoretical upscaling by

59 Battiato and Tartakovsky (2011). Experimental observations documenting the evolution of the

60 pore-scale reaction rate in a Hele-Shaw cell are illustrated by de Anna et al. $(2013,2014)$. These

61 authors show that the spatial distribution of the reaction rates within the pore space is highly non 
62 uniform in space and is characterized by a filamentary structure. The pore-scale dynamics of the

63 process of mixing of the reactants, which is inherently linked to the reaction product, has also

64 been investigated through detailed numerical simulations in various settings (e.g., Tartakovsky et

65 al., 2009; Willingham et al., 2010; Porta et al. 2012b, 2013; Hochstetler and Kitanidis, 2013;

66 Rolle and Kitanidis, 2014; Alhashmi et al., 2015). These studies emphasize the critical

67 importance of a proper model of local mixing for the interpretation of reactive transport

68 processes. Similar conclusions are drawn from column scale experiments where $\mathrm{pH}$-controlled

69 reactions take place (e.g., Jose and Cirpka, 2004; Loyaux-Lawniczak et al., 2012; Edery et al., 70 2015).

71 Several alternative approaches have been proposed with the aim of embedding effects of 72 pore-scale incomplete mixing within Darcy-scale effective reactive transport models (see also 73 the review by Edery et al., 2013). These include particle-based Lagrangian methodologies (e.g.,

74 Edery et al. 2009, 2010; Ding et al., 2013) as well as Eulerian approaches (e.g., Sanchez-Vila et 75 al., 2010; Chiogna and Bellin, 2013; Hochstetler and Kitanidis, 2013). Here we focus on the 76 latter set of approaches as applied to bimolecular fluid-fluid reactions. Continuum-scale 77 approaches typically embed effective formulations, which encode incomplete mixing effects into 78 transport and reaction parameters. These effective formulations typically require model 79 calibration against a set of observations, and can be used to interpret available data. In this 80 context, a series of works (e.g., Sanchez-Vila et al., 2010; Chiogna and Bellin, 2013; Rubio et 81 al., 2008) are focused on the interpretation of the experimental dataset of Gramling et al. (2002).

82 These authors document the spatial distribution of the reaction product concentration within a 83 fully saturated column where the host porous medium is a bead pack. Longitudinal (i.e., along 84 the mean flow direction of flow) profiles of solute concentration are reported for diverse time 
85 levels and transport conditions. A comparison of the results obtained in the above referenced 86 works shows that very different modeling approaches allow interpreting this particular 87 experimental dataset with comparable levels of accuracy. This suggests that the information 88 content associated with the distribution of the reaction product in the system is insufficient for a 89 unique characterization of incomplete mixing through effective continuum-scale approaches.

90 Alhashmi et al. (2015) perform a three-dimensional pore-scale simulation in an attempt to 91 replicate the reactive experiment scenario of Gramling et al. (2002). They show that spreading of 92 the reactants across the medium can be quantified in terms of a time dependent dispersion 93 coefficient. The temporal evolution of longitudinal spreading is different for the two reactants A 94 and B. However, to the best of our knowledge, the ability of effective models to characterize the 95 concentration of the two reactants (A and B) within the regions where they mix and the reactive 96 process takes place has not been tested to date.

97 A mathematical formulation relating pore-scale characteristics and effective reactive 98 transport parameters at a continuum-scale can be derived through formal upscaling 99 methodologies, such as the method of volume averaging (e.g., Whitaker, 1999; Orgogozo et al., 100 2010; Valdes-Parada et al., 2011; Guo et al., 2015). The latter is applied to bimolecular reactive 101 transport by Porta et al. (2012a). These authors show that it is possible to obtain a closed 102 nonlocal formulation of a reaction term associated with incomplete mixing in the presence of of 103 a fast reaction (i.e., large $D a$ values). The assumptions underlying the theoretical analysis of 104 Porta et al. (2012a) have been verified by comparison against direct numerical upscaling of 105 pore-scale simulations performed in simple two-dimensional geometries, i.e., a plane channel 106 (Porta et al., 2012b) and an ordered array of cylinders (Porta et al., 2013). The continuum-scale 107 model proposed by Porta et al. (2012a) is presented in terms of a nonlocal integro-differential 
108 formulation, consistent with a recent formulation obtained by Hansen et al. (2014) starting from

109 the generalized master equation. Note that the formulation proposed by Porta et al. (2012)

110 accounts for the temporal dynamics of the spreading process discussed by Alhashmi et al. (2015)

111 by including a nonlocal in time dispersive transport term.

112 In this work we start from pore-scale numerical simulations of a bimolecular

113 homogeneous and fast reaction. We consider the latter to take place within a two-dimensional

114 pore space, which is generated by randomly placing in space a set of circular grains of uniform

115 diameter. The key target of the work is to test our ability to interpret the continuum-scale results

116 obtained from direct upscaling of pore-scale simulations in such a disordered system through

117 continuum-scale models. The reactive transport setting we analyze is similar to the one

118 considered, e.g., in Gramling et al. (2002), and we model it in terms of an effective one-

119 dimensional unsteady transport process at the continuum-scale. We distinguish two different

120 approaches, depending on the type of information employed to compute the associated model

121 parameters: (1) an approach based on the use of effective models which require calibration

122 against reactive transport features that are typically observed at the continuum scale, e.g., spatial

123 distribution or breakthrough curves of reactants; and (2) an approach based on upscaled models

124 which only require information on pore-space geometry and on the velocity field to predict

125 reactive transport at the continuum-scale.

126 A first goal of this work is to provide the numerical approximation of the nonlocal

127 upscaled model of bimolecular reactive transport derived by Porta et al. (2012a). We then

128 consider three additional effective reactive transport models, i.e., the typical advection dispersion

129 reaction equation, which implicitly assumes complete mixing at pore-scale, and the models

130 proposed by Sanchez-Vila et al. (2010) and Hochstetler and Kitanidis (2013). These models 
131 differ in terms of the formulation employed to characterize the effective reaction parameters.

132 Consistent with typical applications, we estimate these parameters through results obtained from

133 numerical upscaling of pore-scale calculations.

134 Available experimental datasets typically document the evolution of the reaction product

135 in space and time (Gramling et al., 2002; Raje and Kapoor, 2000). Therefore, the majority of

136 previous works dealing with this reactive transport setting are solely based on the interpretation

137 of the space-time evolution of the reaction product. In this work we explicitly consider the model

138 performance also in terms of its ability to capture the dynamics of the concentrations of the two

139 reactants $\mathrm{A}$ and $\mathrm{B}$. This aspect is key in a pre-asymptotic transport regime, where the

140 characterization of transport can be significantly affected by the initial distribution of the

141 reactants in the domain (e.g., Alhashmi et al., 2015). We compare the ability of all these model

142 formulations to interpret the considered reactive transport process and analyze the impact of

143 incorporating diverse types of information in the estimation of model parameters. By doing so,

144 we also identify the critical relevance of specific formats assumed by continuum-scale reactive

145 and transport terms. The results of this work allow quantifying the relevance of nonlocal terms in

146 the reactive transport setting we analyze.

147 The work is organized as follows. Section 2 provides a description of the pore-scale 148 problem setting. Section 3 is devoted to a presentation of the continuum-scale modeling options 149 we implement. In Section 4 we assess the capabilities of the selected continuum-scale models to 150 interpret the pore-scale simulation results. Concluding remarks end the work.

\section{PROBLEM SETTING}

152 We consider the two-dimensional domain $\Omega$ filled by a fully saturated porous medium, 153 consisting of a liquid $\left(\Omega_{l}\right)$ and a solid fraction $\left(\Omega_{s}\right)$. The porous domain is constituted by a 
154 collection of periodic unit cells, $\Omega^{\prime}$. The geometry of the unit cell is generated by the disordered 155 superposition of circular grains of uniform diameter $\hat{w}=0.08 \mathrm{~mm}$ and is characterized by a 156 porosity $\phi \approx 0.6$ (see Figure 1). Note that here and in the following all primed variables are 157 defined within a unit cell and hat-signed variables are dimensional. The liquid and the solid 158 phases share an impermeable boundary surface, $\Gamma_{l s}$. Fluid flow through the pore space is 159 associated with a steady laminar two-dimensional velocity field, $\hat{\mathbf{u}}(\hat{\mathbf{x}})$, which satisfies the Stokes 160 problem. The flow field is driven by a unit pressure gradient along the $x$-direction and a zero 161 pressure gradient along the $y$-direction (Figure 1). The numerical approximation of the velocity 162 field is obtained by the methodology introduced by Bekri et al. (1995) and Coelho et al. (1997).

163 A desired value of average fluid velocity, $\hat{U}$, along the $x$-direction is imposed by multiplying the 164 computed velocity field by an appropriate constant. Figure 1 depicts the dimensionless velocity

165 norm $|\mathbf{u}|=|\hat{\mathbf{u}}| / \hat{U}$. We observe velocity values which are smaller than $\hat{U}$ (i.e., $|\mathbf{u}|<1$ ) throughout 166 most of the domain, values of $|\mathbf{u}|>3$ being mostly concentrated only within a few pore throats. 167 One can clearly identify the presence of regions which are characterized by small velocity values 168 and are poorly connected to the flow preferential path (e.g., regions highlighted in Figure 1). The 169 dimensionless velocities varies in space over a range of values which is comparable to that 170 obtained by flow simulation performed within millimeter-scale imaged rock samples (see e.g., 171 Bijeljic et al., 2013).

172 The external boundary $\Gamma_{\text {ext }}$ of $\Omega_{l}$ can be subdivided into three parts according to the sign 173 of $\hat{\mathbf{u}}(\hat{\mathbf{x}}) \cdot \mathbf{n}_{e}$, i.e., $\Gamma_{\text {in }}\left(\hat{\mathbf{u}}(\hat{\mathbf{x}}) \cdot \mathbf{n}_{e}>0\right), \Gamma_{\text {out }}\left(\hat{\mathbf{u}}(\hat{\mathbf{x}}) \cdot \mathbf{n}_{e}<0\right)$, and $\Gamma_{i m p}\left(\hat{\mathbf{u}}(\hat{\mathbf{x}}) \cdot \mathbf{n}_{e}=0\right), \mathbf{n}_{e}$ being the 174 inward unit vector normal to the boundary. A bimolecular irreversible reaction of the kind A + B 
$175 \rightarrow \mathrm{C}$ takes place in the liquid phase and it is assumed not to affect the velocity field and the pore 176 space geometry. Molecular diffusion is modeled through the standard Fick's law. We employ the

177 following dimensionless formulation of the reactive transport problem (Porta et al., 2013)

178

$$
\frac{\partial c_{i}}{\partial t}+\mathbf{u} \cdot \nabla c_{i}=\frac{1}{P e} \nabla^{2} c_{i} \quad i=D, E
$$

$$
\frac{\partial c_{B}}{\partial t}+\mathbf{u} \cdot \nabla c_{B}=\frac{1}{P e} \nabla^{2} c_{B}-\frac{D a}{P e} c_{B}\left(c_{B}-c_{D}\right)
$$

180 where

$$
\hat{c}_{i}=\hat{c}_{0} c_{i}, \hat{\mathbf{u}}=\hat{U} \mathbf{u}, \hat{t}=t \frac{\hat{\ell}}{\hat{U}}, \hat{\nabla}=\frac{\nabla}{\hat{\ell}}, P e=\frac{\hat{U} \hat{\ell}}{\hat{D}_{m}}, D a=\frac{\hat{k} \hat{c}_{0} \hat{\ell}^{2}}{\hat{D}_{m}}
$$

$182 \hat{c}_{i}$ is concentration, $\hat{c}_{0}$ is a reference concentration; $\hat{k}\left[\mathrm{~m}^{3} /(\mathrm{mol} \mathrm{s})\right]$ is the reaction kinetic

183 constant; $\hat{D}_{m}$ is molecular diffusion $\hat{\ell}$ is a characteristic spatial dimension; and $P e$ and $D a$ are

184 the Péclet and Damköhler numbers, respectively. Note that (1)-(2) are written in terms of the 185 conservative components $c_{D}=c_{B}-c_{A}$ and $c_{E}=c_{B}+c_{C}$. System (1)-(2) can be readily obtained

186 from algebraic recombination of the mass conservation equations written for the three chemical 187 species A, B and C (e.g., Porta et al., 2012a and references therein).

188 We consider a replacement setting: the system is initially saturated by a constant 189 concentration $\hat{c}_{0}$ of species A for $x>0$; species B is introduced continuously in the system for $t$ $190>0$ at a constant concentration $\hat{c}_{0}$ through the boundary $\Gamma_{\text {in }}$. Equations (1)-(2) are then 191 completed by the following boundary and initial conditions

$$
\mathbf{n} \cdot \nabla c_{i}=0 \quad \quad \quad \quad \mathbf{x} \in B, D, E ; \quad \Gamma_{l s}, \Gamma_{\text {imp }}, \Gamma_{\text {out }}
$$

$$
\mathbf{u} \cdot \mathbf{n}_{e} c_{i}(\mathbf{x}, t)+\frac{1}{P e} \mathbf{n}_{e} \cdot \nabla c_{i}(\mathbf{x}, t)=N_{i}, i=B, D, E \quad \mathbf{x} \in \Gamma_{i n}
$$




$$
c_{B}(\mathbf{x}, 0)=0, c_{D}(\mathbf{x}, 0)=-1, c_{E}(\mathbf{x}, 0)=0 \quad \mathbf{x} \in \Omega_{l}, x>0
$$

$$
c_{B}(\mathbf{x}, 0)=1, c_{D}(\mathbf{x}, 0)=1, c_{E}(\mathbf{x}, 0)=1 \quad \mathbf{x} \in \Omega_{l}, x<0
$$

196 A flux boundary conditions is assumed at $\Gamma_{i n}$ so that $c_{B}=c_{D}=c_{E}=1$. Zero flux is imposed on

197 the liquid-solid interface $\Gamma_{l s}$, and $\Gamma_{i m p}$. Note that the boundary $\Gamma_{i n}$ is located at $x=-60$ so that

198 the pore space is saturated with a constant concentration of B at $t=0$ within the first unit cell

199 adjacent to the inlet section (i.e., for $-60<x<0$ ). This choice allows avoiding any influence of 200 the inlet boundary condition on the reactive process at early times.

201 The reactive transport problem is solved within the pore space by means of the particle 202 tracking methodology described by Porta et al. (2012b, 2013) and adapted from routines 203 developed by Bekri et al. (1995) and Debenest et al. (2005) for the pore scale description of 204 reactive transport. We select $\hat{\ell}=\hat{w}=0.08 \mathrm{~mm}$ and set $P e=24.88$ (i.e., average velocity $205 \hat{U}=0.62 \mathrm{~mm} / \mathrm{s}$, Reynolds number $R e=0.05)$ and $D a=1038$. This value of $P e$ is computed by 206 relying on the average fluid velocity and suggests that the process takes place under moderately 207 advection-dominated conditions. We note that the local velocity distribution displays large 208 variations within the considered disordered cell (see Figure 1). The relative strength of the time 209 scales related to transport by advection and diffusion can be evaluated through the local Péclet 210 number $P e_{l o c}(\mathbf{x})=|\hat{\mathbf{u}}| \hat{\ell} / \hat{D}_{m}$ (Porta et al., 2015). One can expect transport to be strongly 211 advection-dominated along the fast channels detected in the system, where the largest values of 212 velocity are about one order of magnitude larger than the average velocity (i.e., $213|\mathbf{u}| \approx 10, P e_{l o c}(\mathbf{x}) \approx 250$ ), while diffusion drives solute transport within the cavities, where the 214 fluid is basically immobile, i.e. $P e_{l o c}(\mathbf{x}) \approx 0$. 
215 The total longitudinal length of the system is $L=1200(\hat{L}=9.6 \mathrm{~cm})$, i.e. we consider a 216 collection of 20 unit cells $\Omega^{\prime}$ along the $x$-direction. Our computations rely on a discretization of 217 the pore space into elementary squares $K$ (pixels) of size $0.02 \mathrm{~mm}$. As in Porta et al. (2013), we 218 consider a unit concentration to be equivalent to a number of particles $N P_{K}=100$ per elementary 219 square. Note that, as a result of these modeling choices, simulating a uniform unit concentration 220 of a single chemical species along the whole computational domain requires a number of 221 approximately $1.7 \times 10^{7}$ particles. This yields reliable local (pixel-scale) concentration values for $222 c>5 \times 10^{-3}$ (Porta et al., 2013, 2012b).

223 Figure 2 shows a snapshot of the spatial distribution of $c_{A}, c_{B}$ and $c_{C}$ within the pore space 224 for $t=448$. Note that reactant $\mathrm{A}$ is initially residing in the system. Positive values of $c_{A}$ are 225 observed for $x<500$ solely within pores which are scarcely accessible to flow, these areas 226 corresponding to regions (cavities) highlighted in Figure 1. The invading reactant B tends to 227 follow the principal pathways, characterized by large velocity values. As a consequence, local 228 concentrations of reactants exhibit significant and sharp variations across relatively small 229 distances. We also observe that reactant concentrations are anti-correlated, i.e., $c_{B}$ is small in 230 pores where concentration of $\mathrm{A}$ is significant and vice versa, due to the occurrence of the fast 231 reaction. This is consistent with previous computational analyses in regular two-dimensional 232 geometries (Porta et al., 2013). The concentration of the reaction product C in Figure 2 displays 233 a peak value at $x \approx 450$. Contributions to $c_{C}$ in the forward tail $(x>500)$ are mainly associated 234 with regions characterized by large velocity, stagnant regions chiefly contributing to the 235 backward tail $(x<400)$. 
237 against which outputs of continuum-scale models are compared. For this purpose we consider 238 section-averaged concentrations in the fluid phase. These are defined as

239

$\bar{c}_{i}(x)=\frac{1}{H \bar{\eta}} \int_{H} c_{i}(x, y) d y ; \quad \bar{\eta}=\frac{1}{H} \int_{H} G(x, y) d y$

240 where $H$ is the total length of the porous medium along $y, \bar{c}_{i}$ is section-averaged concentration of

241 local concentrations $c_{i}$ of chemical species $i=\mathrm{A}, \mathrm{B}$, or $\mathrm{C}$ and $G(x, y)$ is an indicator function, i.e.

$242 G(x, y)=0,1$ respectively for solid grains and fluid.

\section{CONTINUUM-SCALE MODELS}

244 A number of approaches have been proposed to model this reactive problem at the Darcy-

245 scale. In this work we consider three existing effective models together with the upscaled

246 formulation stemming from volume averaging of the system (1)-(2), as provided by Porta et al.

247 (2012a). In Section 3.1 we summarize the salient features of the considered effective models.

248 Section 3.2 recalls the definition of the continuum-scale system resulting from volume averaging

249 of the pore-scale system (1)-(2).

\subsection{Effective models}

251 Effective continuum models of reactive transport are typically formulated through an 252 advection dispersion reaction equation (ADRE)

$253 \quad \frac{\partial C_{i}}{\partial t}=\frac{1}{P e}\left(D^{*}+1\right) \frac{\partial^{2} C_{i}}{\partial x^{2}}-U \frac{\partial C_{i}}{\partial x} \quad i=D, E$

$254 \quad \frac{\partial C_{B}}{\partial \hat{t}}=\frac{1}{P e}\left(D^{*}+1\right) \frac{\partial^{2} C_{B}}{\partial x^{2}}-U \frac{\partial C_{B}}{\partial x}-K_{e f f} C_{B}\left(C_{B}-C_{D}\right)$ 
255 where $D^{*}=\hat{D}^{*} / \hat{D}_{m}$ is the dimensionless longitudinal dispersion coefficient, $K_{\text {eff }}$ is an effective 256 reaction constant, $C_{i}(i=B, D, E)$ are continuum-scale concentrations. We consider in the 257 following three models for $K_{\text {eff }}$ proposed in the literature:

$$
K_{e f f}=\frac{D a}{P e}
$$

- Model 2 (Sanchez Vila et al., 2010):

$$
K_{e f f}=\beta \frac{D a}{P e} ; \beta=\frac{\hat{\beta}_{0} \hat{t}^{-m}}{\hat{k}}
$$

$$
\text { where } \hat{\beta}_{0} \text { and } m \text { are parameters to be estimated through model calibration. }
$$

- $\quad$ Model 3 (Hochstetler and Kitanidis, 2013):

$$
K_{e f f}=E_{F} \frac{D a}{P e} ; E_{F}=\frac{\gamma \lambda}{D a+\lambda}
$$
where $E_{F}$ is the reaction effectiveness factor, $\gamma$ and $\lambda$ being model parameters. Model 1 implies that the same reaction constant can be employed at the pore and continuum267 scales, i.e. reactants are assumed to be completely mixed at the pore-scale. Models 2 and 3 268 account for the effect of incomplete mixing of the reactants through the effective reaction 269 parameters (12)-(13). The definitions in (12)-(13) are motivated by previous literature 270 approaches to model conservative and reactive transport. Sanchez-Vila et al. (2010) introduce 271 (12) based on the rate-limited mass transfer process observed by Haggerty et al. (2004). 272 Hochstetler and Kitanidis (2013) introduce the reaction effectiveness factor $E_{F}$ (13) following 273 the definition of the segregation intensity introduced by Kapoor et al. (1997). 
Porta et al. (2012a) perform an upscaling of (1)-(2) through the volume averaging

276 method (Whitaker, 1999). Due to the nature of the scenario we consider, we recall here the

277 corresponding one-dimensional formulation, which is labeled as model 4 in the following. A key

278 target of the volume averaging analysis is to obtain a closure formulation in terms of the intrinsic

279 volume averaged concentration

$280\left\langle c_{i}\right\rangle^{l}=\frac{1}{\phi V(\mathbf{x})} \int_{V} c_{i}(\mathbf{x}) d V$

281 where $V(\mathbf{x})$ is a volume of porous medium within $\Omega$. The volume averaged formulation for the 282 conservative species reads

$283 \frac{\partial\left\langle c_{i}\right\rangle^{l}}{\partial t}=-\langle u\rangle^{l} \frac{\partial\left\langle c_{i}\right\rangle^{l}}{\partial x}+\frac{1}{P e} \frac{\partial}{\partial x}\left[\left(1+D_{U}\right) * \frac{\partial}{\partial t}\left(\frac{\partial\left\langle c_{i}\right\rangle^{l}}{\partial x}\right)\right] \quad i=D, E$

284 where

$285 \quad D_{U} * \frac{\partial}{\partial t}\left(\frac{\partial\left\langle c_{i}\right\rangle^{l}}{\partial x}\right)=\int_{0}^{t} D_{U}(t-\tau) \frac{\partial}{\partial \tau} \frac{\partial\left\langle c_{i}\right\rangle^{l}}{\partial x} d \tau ; \quad D_{U}=\frac{1}{V} \int_{V_{l s}} b n_{x} d A+\langle\tilde{u} b\rangle^{l}$

286 is the dispersion coefficient, $\tilde{u}=u-\langle u\rangle^{l}$ is a spatial fluctuation (or deviation) of the velocity in

287 the $x$-direction within the pore space, and $b$ is a closure variable. Equations (15)-(16) are based 288 on the following unsteady closure relationship (Moyne, 1997; Chastanet and Wood, 2008)

289

$$
\tilde{c}_{i}=c_{i}-\left\langle c_{i}\right\rangle^{l}=b\left(\mathbf{x}^{\prime}, t\right) * \frac{\partial}{\partial t} \frac{\partial\left\langle c_{i}\right\rangle^{l}}{\partial x}
$$


290 where $\tilde{c}_{i}=c_{i}-\left\langle c_{i}\right\rangle^{l}(i=\mathrm{D}, \mathrm{E})$ is a spatial fluctuation of the concentration about the mean. The

291 medium we consider is characterized by a periodic structure and the closure variable $b$ can be

292 computed within a unit cell. The following differential problem is satisfied by $b$

$293 \quad \frac{\partial b}{\partial t}+(\mathbf{u} \nabla b+\tilde{u})=\frac{1}{P e} \nabla^{2} b \quad \quad \mathbf{x}^{\prime} \in \Omega_{l}^{\prime}, t>0$

$294 \mathbf{n} \cdot \nabla b=n_{x}$

$\mathbf{x}^{\prime} \in \Gamma_{l s}^{\prime}, t>0$

$295 b\left(\mathbf{x}^{\prime}, 0\right)=0$

$\mathbf{x}^{\prime} \in \Omega_{l}^{\prime}, t=0$

296 Here, we have considered the unit cell reference system $\mathbf{x}^{\prime}$, with the corresponding liquid $\Omega_{l}^{\prime}$

297 domain and the liquid-solid surface $\Gamma_{l s}^{\prime}$. The format of the transport equation for the reactive

298 species B depends on the relative importance of $D a$ as compared to Pe. Here, we consider $D a>>$

299 Pe so that (Porta et al., 2012a)

$300 \frac{\partial\left\langle c_{B}\right\rangle^{l}}{\partial t}+\langle u\rangle^{l} \frac{\partial\left\langle c_{B}\right\rangle^{l}}{\partial x}=\frac{1}{P e} \frac{\partial}{\partial x}\left[\frac{\partial\left\langle c_{B}\right\rangle^{l}}{\partial x}+M\left(D_{U}-1\right) * \frac{\partial}{\partial t}\left(\frac{\partial\left\langle c_{D}\right\rangle^{l}}{\partial x}\right)\right]-\frac{D a}{P e}\left(R_{1}+R_{2}\right)$

301 where

$302 \quad R_{1}=\left\langle c_{B}\right\rangle^{l}\left(\left\langle c_{B}\right\rangle^{l}-\left\langle c_{D}\right\rangle^{l}\right)$

$303 \quad R_{2}=M(M-1)\left\langle\left(b * \frac{\partial}{\partial t} \frac{\partial\left\langle c_{D}\right\rangle^{l}}{\partial x}\right)^{2}\right\rangle_{l}$

304 and $M=\frac{\left\langle c_{B}\right\rangle^{l}}{\left\langle c_{B}\right\rangle^{l}+\left\langle c_{A}\right\rangle^{l}}=\frac{\left\langle c_{B}\right\rangle^{l}}{2\left\langle c_{B}\right\rangle^{l}-\left\langle c_{D}\right\rangle^{l}}$. The reaction terms (22)-(23) stem from volume

305 averaging of the pore-scale reaction term

$306\left\langle c_{B}\left(c_{B}-c_{D}\right)\right\rangle^{l} \approx\left\langle c_{B}\right\rangle^{l}\left(\left\langle c_{B}\right\rangle^{l}-\left\langle c_{D}\right\rangle^{l}\right)+\left\langle\tilde{c}_{B}\left(\tilde{c}_{B}-\tilde{c}_{D}\right)\right\rangle^{l}$ 
$308 \quad \tilde{c}_{B} \approx \tilde{c}_{D} \frac{\left\langle c_{B}\right\rangle^{l}}{2\left\langle c_{B}\right\rangle^{l}-\left\langle c_{D}\right\rangle^{l}}=M \tilde{c}_{D}=M b * \frac{\partial}{\partial t} \frac{\partial\left\langle c_{D}\right\rangle^{l}}{\partial x}$

309 Substitution of (25) in (24) leads to $R_{2} \approx\left\langle\tilde{c}_{B}\left(\tilde{c}_{B}-\tilde{c}_{D}\right)\right\rangle^{l}$. We refer to the formulation (15)-(21) as

310 model 4 in Section 4 for simplicity. The parameters appearing in (15)-(21) can be fully

311 characterized from pore-scale geometry and velocity distribution upon solving the system (18)-

312 (20). We do so by implementing a finite element formulation of (18)-(20) in the FreeFEM++

313 environment (Hecht et al., 2012). We discretize the solution in space through a stabilized

314 streamline diffusion finite element formulation while employing a first order implicit backward

315 Euler finite difference scheme in time. We compute the solution by employing a structured grid

316 formed by approximately $10^{5}$ triangular elements.

317 Figure 3 depicts the time evolution of the coefficients $D_{U}(16)$ and of the quantity

$318 \quad B_{2}(t)=\left\langle b\left(\mathbf{x}^{\prime}, t\right)^{2}\right\rangle_{l}$

319 We select $B_{2}$ as a measure of the nonlocal reaction term (23), i.e., the localized counterpart of 320 (23) reads

$321 \quad R_{2, L O C}=M(M-1) B_{2}(t)\left(\frac{\partial\left\langle c_{D}\right\rangle^{l}}{\partial x}\right)^{2}$

322 We observe that both $D_{U}$ and $B_{2}$ vary over a wide range of values and are always increasing 323 within the considered time window. This result evidences that the reactive transport process we 324 observe takes place within a pre-asymptotic regime. Therefore, the nonlocal terms embedded in 325 (15)-(21) can be expected to play a relevant role in the reactive system characterization. 
The numerical solution of the upscaled system requires the discretization of the integro-

327 differential terms appearing in (15)-(21). We employ a trapezoid integration rule to discretize the

328 integral terms (16) and (23) in the time domain. System (15)-(21) is approximated through a

329 fixed space and time discretization. We select a time step $\Delta t=2$ and a spatial discretization step

$330 \Delta x=1$. We numerically verified through a grid convergence analysis that the results do not

331 depend on the spatial discretization and time step size (details not shown). We also verify that

332 the results of the nonlocal solution reproduce those of the corresponding local formulation when

333 parameters are constant in time. The computational time associated with a run of the nonlocal

334 model 4 is approximately $10^{5} \mathrm{~s}$ (28 hours) on a processor Intel(R) Pentium(R) III Xeon processor

$3352.83 \mathrm{GHz}$. For comparison, note that the numerical solution of the effective models (9)-(10)

336 requires approximately $10^{3} \mathrm{~s}$ on the same processor.

\section{COMPARATIVE ASSESSEMENT OF CONTINUUM-SCALE MODELS}

In this section we discuss the ability of the continuum-scale models introduced in Section

3393 to reproduce the results obtained by pore-scale simulation of the reactive transport process.

340 As anticipated in the Introduction, we compare two classes of models which are

341 distinguished on the basis of the information sources employed to characterize the model

342 parameters and are associated with diverse levels of complexity. Models 1-3 have a simple

343 structure, but their effective parameters are estimated through model calibration, i.e. they

344 incorporate information on the output concentration profiles. On the other hand, model 4 is

345 characterized by a complex structure, and the related parameters are estimated relying solely on

346 pore-scale information, i.e. no model calibration is required. Our model comparison aims at

347 assessing the impact of the critical differences amongst the considered continuum-scale models

348 on the ability of the models to reproduce our pore-scale reference solution. These key differences 
can be classified as illustrated in the following. With reference to transport, model 4 is nonlocal

350 in time and allows accounting for non-Fickian dispersion effects, as opposed to models 1-3

351 which are all based on an assumed Fickian dispersion process. With reference to the way 352 reaction is embedded in the model, model 4 entails a space and time dependent nonlocal 353 formulation of the reaction rate constant; model 2 embeds a time dependent effective reaction 354 parameter $K_{\text {eff }}$ through (12); while models 1 and 3 employ a constant effective reaction 355 coefficient, $K_{\text {eff }}$.

356 In the following, we start by discussing the characterization of the parameters of models

357 1-3, which is performed through calibration against concentration profiles extracted from the 358 reference pore-scale simulation. We then compare the results of the set of the four continuum359 scale models illustrated in Section 3 against (section-averaged) pore-scale simulation results.

\subsection{Calibration of the effective models}

The characterization of the effective models (9)-(13) (models 1-3) is here performed 362 through the estimation of the embedded effective parameters, performed via model calibration.

363 The model calibration parameters we consider are listed in Table 1 for each of the three models.

364 The longitudinal dispersion coefficient is considered as a calibration parameter for all 365 three models. This choice is motivated from results of previous studies focusing on the 366 interpretation of the dataset of Gramling et al. (2002). In this context, Alhashmi et al. (2015) 367 demonstrate through numerical pore-scale simulation that transport in the system is still in a pre368 asymptotic stage. Otherwise, results by Sanchez-Vila et al. (2010) show that a Fickian model can 369 be employed to interpret the same reactive transport dataset up to a reasonable accuracy by 370 including the dispersion coefficient as a parameter to be estimated through model calibration 371 against the reactive transport data. 
373 with the respective reaction models, i.e. $\hat{\beta}_{0}$ and $m$ in (12) for model $2, \gamma$ and $\lambda$ in (13) for model

3743 . Given the structure of the reactive term in (13), we note that infinite combinations of $\gamma, \lambda$ can

375 lead to the same effective reaction constant for a given $D a$. This implies that joint estimation of $376 \gamma$ and $\lambda$ is possible only when calibration data associated with diverse values of $D a$ are 377 available. Since we consider here a unique value of $D a$, we follow the indication of Hochstetler 378 and Kitanidis (2013) and set $\gamma=1$ while estimating $\lambda$.

379 Our aim is here to study the performance of these models in the presence of different sets 380 of calibration data. We consider two sets of such data, i.e., concentration profiles of the invading 381 reactant $\mathrm{B}\left(\right.$ dataset $\left.\bar{c}_{B i}^{*}\right)$ and of the reaction product $\mathrm{C}$ (dataset $\bar{c}_{C i}^{*}$ ) at a fixed dimensionless time,

$382(t=448)$. We select this time level because it is the longest time considered in the pore-scale 383 simulation. We then calibrate all three models by employing separately the two datasets. Entries 384 of the calibration datasets are concentrations $\bar{c}_{B i}^{*}\left(i=1 \ldots N_{D B}\right)$, and $\bar{c}_{C i}^{*}\left(i=1 \ldots N_{D C}\right), N_{D B}$ 385 and $N_{D C}$ indicating the number of data included in the datasets $\bar{c}_{B i}^{*}$ and $\bar{c}_{C i}^{*}$, respectively. We 386 select values $\bar{c}_{C i}^{*}$ as

$387 \quad \bar{c}_{C i}^{*}=\frac{1}{L^{\prime}} \int_{L^{\prime} \cdot(i-1)}^{L^{\prime} \cdot i} \bar{c}_{C} d x \quad i=1, \ldots, 20$

388 where $L^{\prime}=60$ is the length of the unit cell $\Omega^{\prime}$ along the $x$-direction. In other words, we consider

389 the 20 unit cells constituting the total length $L$ of the porous domain and identify $\bar{c}_{C i}^{*}$ as the 390 spatial average of the pore-scale concentrations of the reaction product $c_{C}$ computed within each 391 of the unit cells (i.e, we set $N_{D C}=20$ ). 
393 concentration in the liquid phase

$394 \quad \bar{c}_{B i}^{*}=\frac{1}{L^{\prime}} \int_{L^{\prime} \cdot(i-1)}^{L^{\prime} \cdot i} \bar{c}_{B} d x \quad i=1, \ldots, 8$

395 Note that positive values of $c_{B}$ are observed only for $x<500$, i.e., in the first 8 unit cells. We

396 augment the entries in this calibration dataset by considering additional concentration values at

397 locations close to the reaction front $(x \approx 450)$, because one of our goals is the characterization of

398 the parameters of the incomplete mixing models which are expected to influence concentration

399 values at such locations. As such, dataset $\bar{c}_{B i}^{*}$ includes a total of 16 data which are distributed

400 along the length of the porous domain $\left(N_{D B}=16\right)$. The spatial distributions of the calibration

401 data $\bar{c}_{B i}^{*}$ and $\bar{c}_{C i}^{*}$ are depicted in Figure $4 \mathrm{a}$ and $4 \mathrm{~d}$, respectively.

402 Model parameters are estimated either through the datasets $\bar{c}_{B i}^{*}$ or $\bar{c}_{C i}^{*}$ by considering the

403 following metrics

$404 \quad J_{C}=\frac{1}{N_{D C}} \sum_{i=1}^{N_{D C}}\left[\bar{c}_{C i}^{*}-C_{C}\left(x_{i}\right)\right]^{2}$

$405 \quad J_{B}=\frac{1}{N_{D B}} \sum_{i=1}^{N_{D B}}\left[\bar{c}_{B i}^{*}-C_{B}\left(x_{i}\right)\right]^{2}$

406 where $C_{J}\left(x_{i}\right)(J=B, C)$ is the continuum-scale model output at location $x_{i}$ corresponding to

407 the location of data $\bar{c}_{B i}^{*}, \bar{c}_{C i}^{*}$. We employ criteria (30)-(31) in a maximum likelihood framework

408 (Carrera and Neuman, 1986), where we assume the covariance matrix associated with

409 measurements error to be equal to a diagonal matrix with constant entries. This enables us to

410 compute the covariance matrix of the estimation error 
$411 \mathbf{Q}_{k, C_{j}}=\sigma_{k, C_{j}}^{2}\left(\mathbf{J}_{k, C_{j}}^{T} \mathbf{J}_{k, C_{j}}\right)^{-1} \quad k=1,2,3 ; j=B, C$

412 where indices $k$ and $j$ respectively indicate the model and the dataset employed for model 413 calibration, $\sigma_{k, C_{j}}^{2}=J_{j, m i n} / N_{D j}$ is the estimated model error, $\mathbf{J}_{k, j}$ is the Jacobian matrix whose

414 entries are the derivatives of the output state variables $C_{j}$ with respect to the parameters of model

$415 k$. The diagonal entries of matrix (32) quantify the uncertainty $\sigma_{p}^{2}$ associated with the ML 416 estimate of model parameter $p$.

417 Table 1 lists the values of the estimated parameters and the related standard deviation $\sigma_{p}$, 418 together with the minimum values of (30)-(31) (respectively denoted as $J_{C \min }$ and $J_{B \min }$ ) obtained 419 through calibration against the two types of data $\bar{c}_{B i}^{*}$ and $\bar{c}_{C i}^{*}$ for each of the three effective 420 models (9)-(13). The calibrated model results are compared in Figure 4 against the calibration 421 data and the complete profile of section-averaged concentrations $\bar{c}_{B}(x)$ and $\bar{c}_{C}(x)$ rendered by 422 pore-scale simulation at $t=448$. Models 2 and 3 lead to smaller values of $J_{C \min }$ than model 1. 423 This result is consistent with previous analysis (Sanchez-Vila et al., 2010; Hochstetler and 424 Kitanidis, 2013) and supports the idea that effective models 2-3 can interpret the distribution of 425 the reaction product in the reactive transport setting we analyze in the presence of pore-scale 426 incomplete mixing of reactants. We also observe that the values of $J_{B m i n}$ are similar for models 1

427 and 2 and $J_{B \min }$ is largest for model 3 . We note that values of standard deviation $\sigma_{p}$ associated 428 with the estimates of the dispersion parameters are about 5-10\% of the corresponding ML 429 estimate for all three models and for the two considered datasets, thus resulting in relatively 430 small coefficients of variation. These results suggest that the data convey appropriate 431 information to characterize the longitudinal dispersion coefficients. However, note that the 
432 estimated longitudinal dispersion coefficients are significantly different across models and/or

433 depending on the type pf calibration data employed. Model calibrations based solely on $\bar{c}_{B i}^{*}$ yield

434 estimated longitudinal dispersions which are considerably larger than those based on $\bar{c}_{C i}^{*}$ (see

435 Table 1). This leads to an increased spreading of the reaction product when these models are

436 calibrated against $\bar{c}_{B i}^{*}$ as opposed to what can be obtained by employing $\bar{c}_{C i}^{*}$ as a calibration

437 dataset (see Figure $4 b$ and d).

438 The estimate of parameter $m$ in model 2 rendered by calibration against $\bar{c}_{C i}^{*}$ is consistent

439 with the value obtained by Sanchez-Vila et al. (2010) by using the same type of information.

440 Note that Table 1 lists $\hat{\beta}_{0}$ in dimensional units $\left[\mathrm{m}^{3} \mathrm{~mol}^{-1} \mathrm{~s}^{m-1}\right]$. Given that the dimensional units of

441 this parameter depend on the specific value assumed by the exponent $m$, the values of $\hat{\beta}_{0}$

442 obtained in Table 1 are hardly comparable to those obtained in Sanchez-Vila et al. (2010). The

443 best estimate obtained for parameter $\lambda$ in model 3 is of the same order of magnitude of the one

444 obtained by Hochstestler and Kitanidis (2013) when $\bar{c}_{C i}^{*}$ data are employed. In general, we

445 observe that the estimated values of the effective reaction parameters embedded in models 2 and

4463 largely depend on the type of information available for calibration.

$447 \quad$ Figure $4 \mathrm{~d}$ shows that the peak of concentration $C_{C}$ rendered by models 2 and 3 is smaller

448 than that associated with model 1 when $\bar{c}_{C i}^{*}$ is considered for calibration. Otherwise, the three

449 effective models yield the same concentration peak of $C_{C}$ when their parameters are estimated

450 against $\bar{c}_{B i}^{*}$ (see Figure 4b). Previous studies (e.g., Sanchez-Vila et al., 2010; Chiogna and Bellin,

451 2014) show that the evolution of the peak concentration is typically linked to incomplete mixing 
452 of the reactant at pore-scale. We further note from Table 1 that the standard deviations related to 453 the effective reaction parameters embedded in models 2 (i.e, $\hat{\beta}_{0}$ and $m$ ) and 3 (i.e, $\lambda$ ) are much 454 larger when calibration is performed through dataset $\bar{c}_{B i}^{*}$ than by relying on $\bar{c}_{C i}^{*}$. Therefore, 455 results in Figure $4 \mathrm{~b}$ and $4 \mathrm{~d}$ and Table 1 suggest that an accurate estimation of the incomplete 456 mixing parameters embedded in models 2 and 3 requires information on the reaction product 457 concentration. This result can be explained upon observing that the output concentration $C_{B}$ 458 rendered by the investigated continuum models attains very small values close to the reaction 459 front. Large variations of the incomplete mixing parameters induce modest variations of $C_{B}$.

460 Otherwise, the peak concentration of $C_{C}$ is very sensitive to variations of these reaction model 461 parameters, as previously shown by Sanchez-Vila et al. (2010), Chiogna and Bellin (2013), and 462 Ciriello et al. (2015). Therefore, model calibration against $\bar{c}_{C i}^{*}$ yields a reduced uncertainty for 463 the reaction parameters which represent incomplete mixing, as compared to results obtained 464 through calibration based on $\bar{c}_{B i}^{*}$ observations.

\subsection{Model comparison}

In this Section we aim at assessing the performance of the diverse effective and upscaled

467 models in reproducing the pore-scale simulation results, which represent our reference solution.

468 We compare in our discussion the results obtained through calibration of the effective 469 models 1-3 against the dataset $\bar{c}_{C i}^{*}$ (see Section 4.1) and the prediction yielded by model 4 on the 470 basis of pore-scale information. We focus on a comparison of the longitudinal profiles of 471 concentrations of the three chemical species as rendered by pore- and continuum-scale models 472 and quantify model performance through some global indicators. 
Figures 5a-f depict a comparison between the concentration profiles rendered by the

474 numerical solution of the continuum-scale models and section-averaged concentration obtained

475 through the pore-scale simulation for solutes A (Figure 5a-b), B (Figure 5c-d) and C (Figure 5e-

$4764 \mathrm{f}$ ) at two selected (dimensionless) times, i.e., $t=74$ (Figure 5a,c,e) and $t=448$ (Figure 5b,d,f).

477 As time advances, B displaces A and the two reactants mainly mix in a limited region around the

478 the interface between $\mathrm{B}$ and $\mathrm{A}$ (i.e., the reaction front), which migrates in the system by

479 advection. Owing to our choice of dimensionless space-time reference, the reaction front is

480 found at locations $x \approx t$ for each considered time level. The backward tail of $\bar{c}_{A}$ in our reference

481 pore-scale solution displays an oscillatory behavior characterized by isolated localized peaks (see

482 Figures 5a-b, respectively for $x<70, x<400)$. These oscillations are related to the investigated

483 pore structure, i.e. they are chiefly due to the presence of cavities in the porous domain where the

484 solute is trapped for long times (see also Figures 1-2). As time progresses, the reaction front

485 advances in the porous domain by advection and new immobile (or low velocity) zones with $\bar{c}_{A}$

$486>0$ remain isolated and surrounded by the invading solute B (see Figure 2). Thus, the number of

487 localized concentration peaks in the profile of $\bar{c}_{A}$ increases with time. The effect of these low-

488 velocity zones is also detected on $\bar{c}_{B}$ and $\bar{c}_{C}$ profiles (Figures $5 \mathrm{c}-\mathrm{d}$ and e-f). The section-

489 averaged concentrations of reactants B and A show an anti-correlated behavior, i.e. sharp

490 increases of A concentrations correspond to localized declines of B concentrations, consistent

491 with the pore-scale spatial distributions depicted in Figure 2. The presence of cavities (poorly

492 connected and almost immobile zones) results in localized high concentration peaks that are also

493 visible in the profiles of the reaction product $\mathrm{C}$, which is delayed in low velocity regions. We

494 note that this phenomenon is not observed when considering experimental measurements and 
495 numerical simulation of the same reactive process within three-dimensional regular porous 496 media, e.g., glass beads packing (Gramling et al., 2002; Alhashmi et al., 2015). The considered 497 disordered two-dimensional porous medium includes poorly connected and essentially stagnant 498 regions, giving rise to the local accumulations of species $\mathrm{A}$ and $\mathrm{C}$ observed in Figure 5.

499 All continuum-scale models considered in this study fail to reproduce trapping of 500 concentration of species A within immobile regions (see Figure 5b). To quantify the relevance of 501 this inaccuracy, we consider the temporal evolution of $R C_{A}$, the residual average concentration 502 of $\mathrm{A}$, in a subregion of our computational domain

$503 \quad R C_{A}(t)=\frac{1}{X_{M}} \int_{0}^{X_{M}} C_{A}(x, t) d x$

$504 X_{M}<L$ identifying the longitudinal size of a given subdomain we consider. The time evolution of $505 R C_{A}$ enables us to evaluate the residence time of reactant $\mathrm{A}$ in the system. As an example,

506 Figure 6a depicts the evolution of $R C_{A}$ when $X_{M}=300$, i.e. we compute $R C_{A}$ within a domain

507 segment of longitudinal length corresponding to five unit cells. Since we expect the reaction

508 front to approach the location $X_{M}$ for $t=X_{M}$, selecting $X_{M}=300$ enables us to characterize the 509 temporal dynamics of $R C_{A}$ before and after the main reaction front breaks through location $X_{M}$ 510 within the time window considered in our pore-scale simulation. We observe that the pore-scale 511 simulation yields what appears to be a two-stage evolution of the residual mass of A. This 512 feature is identified by a remarkable change in the slope of $R C_{A}(t)$ which takes place at $t \approx 300$,

513 i.e. for $t \approx X_{M}$. For $t<300$, the average concentration of A progressively decreases to a value 514 approximately equal to $10^{-2}$. For $t>300$, the concentration of A decreases at a lower rate as 515 compared to the above mentioned first stage. In this second stage, the residual mass of A can be 
516 found within system cavities and smoothly decreases in time due to reaction. We observe that all

517 four continuum-scale models reproduce almost exactly the (section-averaged) pore-scale results

518 up to a time $t \approx 250$, the evolution of $R C_{A}$ in the second stage ( $t>300$ ) being misrepresented by

519 all investigated continuum-scale models. Results in Figure 5a-b and 6a suggest that considering

520 (a) effective reaction terms which model incomplete mixing effects (as in models 2-3) and (b)

521 time nonlocalities in both transport and reaction terms (as in model 4) has virtually no effect in

522 improving our ability to reproduce the delayed mass of the reactant A within poorly connected

523 cavities. Figure 6a suggests that all four considered continuum-scale models allow reproducing

524 the residual concentration of the reactant $\mathrm{A}$ in the system with comparable accuracy, i.e. up to 525 values of $0.03-0.05$ (3-5\% of the initial concentration of A).

526 The reference longitudinal profiles of the concentration of the invading reactant, $\bar{c}_{B}$, are

527 well predicted by model 4 for both considered time levels (see Figure $5 \mathrm{c}-\mathrm{d}$ ). The effective 528 models $1-3$ yield $C_{B}=1$ for $x<200$ and $t=448$, the solution associated with model 4 tending 529 smoothly to unity at $x=0$ and closely following the trend of the pore-scale results (see Figure $5305 \mathrm{~d})$. Insets in Figure 5c-d depict profiles of the concentration of the $\mathrm{B}$ reactant in semi 531 logarithmic scale. Model 4 reproduces closely the forward tail of the invading reactant, which is 532 mostly affected by the reactive process. Values of $\bar{c}_{B}$ at locations where $\bar{c}_{B}<0.1$ are 533 overestimated by models $2-3$ and underestimated by model 1 . This result suggests that the 534 volume averaged formulation (15)-(21) (i.e., model 4) leads to an improved continuum-scale 535 representation of mixing at the reactive front, as compared to models 2 and 3, which however 536 provide a reasonably accurate interpretation of the spatial distribution of the concentration of the 537 reaction product $\mathrm{C}$ (see Figure $3 \mathrm{~d}$ ). 
539 corresponding to the solute breakthrough curve $\left(B T C_{B}\right)$. The latter is defined as

$540 \quad B T C_{B}(t)=C_{B}\left(X_{M}, t\right)$ for continuum-scale models and as

$541 \quad \operatorname{BTC}_{B}(t)=\frac{\int_{H} c_{B} u d y}{\int_{H} u d y}$

542 when calculations are performed from pore-scale data. Figure $6 \mathrm{~b}$ depicts the temporal evolution

543 of the breakthrough curve $\left(B T C_{B}\right)$ of $\mathrm{B}$ at position $x=X_{M}=300$. We observe that model 4

544 reproduces the pore-scale results more closely than the remaining three effective models. This

545 result is consistent with longitudinal concentration profiles in Figure 5c-d. The observed

546 differences amongst the models can be explained by considering that model 4 accounts for non-

547 Fickian transport through the nonlocal term (16), while models 1-3 consider a Fickian dispersion

548 model. Moreover, the volume-averaged (upscaled) reaction term (22)-(23) renders an improved

549 representation of the mixing of the reactants close to the reactive front as compared to the

550 effective reaction terms embedded in models 2 and 3. This is suggested by the close agreement

551 between the results rendered by model 4 and their pore-scale counterparts for low concentration

552 values. As a consequence, Figure $6 \mathrm{~b}$ shows that, contrary to the results given by the effective

553 models 1-3, consideration of nonlocal transport effects and of the upscaled reactive terms

554 improves our ability to accurately quantify the arrival times of a reactive solute B at a given

555 location in the system.

556 Figure 5e-f depicts the comparison between the longitudinal profiles of concentration of

557 the reaction product $\mathrm{C}$ rendered by the different models and the reference pore-scale solution.

558 These results are complemented by the temporal evolution of the spatial moments of the reaction 
559 product concentration profiles (Figure 7). Figure 7a shows the temporal evolution of the total

560 mass of $\mathrm{C}$ in the systems, i.e., the zero order moment of the concentration profile

$561 \quad M_{O C}(t)=\int_{L} C_{C}(x, t) d x$

562 Figure $7 b-c$ display the evolution of the spreading

$563 \quad \sigma_{C}(t)=\sqrt{\frac{\int_{L} C_{C}(x, t)\left(x-x_{C}\right)^{2} d x}{M_{0 C}}} ; \quad x_{C}(t)=\frac{\int_{L} C_{C}(x, t) x d x}{M_{0 C}}$

564 and of the skewness

$565 \quad \gamma_{C}(t)=\int_{L} C_{C}(x, t)\left(\frac{x-x_{C}(t)}{\sigma_{C}(t)}\right)^{3} d x$

566 of the concentration of the reaction product. For the pore-scale simulation, quantities (35)-(37)

567 are computed by replacing $C_{C}(x, t)$ in the corresponding expression with the section-averaged

568 concentration $\bar{c}_{C}(x, t)$.

569 Figure 7a reveals that effective models 1-3 lead to an overestimation of the total mass of

$570 \mathrm{C}$ for early times. These three models yield modest errors for long times $(t>300)$. This is

571 consistent with the observation that the parameters of models 1-3 are estimated through the

572 concentration of $\mathrm{C}$ at $t=448$. Model 4 reproduces accurately the total mass of $\mathrm{C}$ for early times,

573 while leading to a slight underestimation of the reference result for $t>200$. All four continuum-

574 scale models underestimate the total reaction product by less than $10 \%$ at time $t=448$.

575 Figure $7 \mathrm{~b}$ depicts the temporal evolution of the spreading of the reaction product $\mathrm{C}$, as 576 quantified through (36), and shows that the longitudinal spreading of the pore-scale 577 concentration $c_{C}$ is underestimated by about $20 \%$ when considering models 1-3, model 4 
578 underestimating it only by $10 \%$. Comparison of the longitudinal profiles reveals that the

579 inaccuracy associated with model 4 is due to an underestimation of spreading in the forward tail

580 (see Figure 5f, $x>450$ ). This may be explained by $(i)$ the modeling error embedded in the

581 approximations underlying the volume averaging procedure, and (ii) numerical inaccuracies

582 related to the approximation of the closure problem (18)-(20) and of the integro-differential

583 terms appearing in (15)-(21). With reference to the latter point, we note that while in this work

584 we implement a standard numerical solver, the development of more sophisticated techniques for

585 the treatment of integro-differential terms might improve the global performance of model 4. A

586 detailed analysis of this aspect is beyond the scope of this contribution. The effective models 1-3

587 underestimate spreading and do not provide a satisfactory interpretation of delayed

588 concentrations of the reaction product $\mathrm{C}$, even as these are explicitly considered in the model

589 calibration procedure (see Figure 5f).

$590 \quad$ Pore-scale results display some early time oscillations of the skewness coefficient (Figure

591 7c). The latter attains a maximum value of 0.05 at $t=50$. After this time the value of $\gamma_{C}$

592 associated with pore-scale concentration shows a sustained decrease until it attains negative

593 values for $t>100$. This result is consistent with the asymmetric pattern observed in Figure 5e-f.

594 In particular, Figure 5f shows that for $t=448$ the backward tail $(x>450)$ is characterized by a

595 smaller average (spatial) gradient than the forward one $(x<450)$. The continuum models $1-3$

596 yield a positive value of $\gamma_{C}$ within the whole considered time window. The non-negligible

597 positive value of the skewness observed at early times for models 1-3 is due to the prescribed set

598 of initial and boundary conditions. We observe that $\gamma_{C}$ tends to zero for long times when we

599 consider the effective models $1-3$, i.e. the spatial distribution of $C_{C}$ tends to become symmetric 
600 around the peak, consistent with the structure of the implied Fickian dispersion picture (see also

601 Figure 5f). Otherwise, results of the nonlocal model 4 are characterized by a negative skewness

602 within most of the considered observation time frame. Even as model 4 underestimates the

603 values of $\gamma_{C}$ rendered by pore-scale simulation, it allows capturing the left tailed behavior of $C_{C}$

604 . This result is associated with the two features which distinguish model 4 with respect to the

605 effective models 1-3, i.e., ( $i$ ) the nonlocal nature of the embedded dispersive transport term, and

606 (ii) the dependence of the reaction term on space and time.

Results of Figure 7 suggest that considering a non-Fickian dispersion model is critical to

608 capture the evolution in time of spreading and of the asymmetry displayed by the longitudinal

609 distribution of the reaction product. This result is consistent with the findings of Edery et al.

$610(2009,2010)$ and Alhashmi et al. (2015). We note that Figure 7a suggests that models based on a

611 Fickian model assumption can interpret the time evolution of the total reaction product mass

612 (corresponding to $M_{O C}$ ) with a reasonable accuracy, even under a pre-asymptotic transport

613 regime, as previously shown by Sanchez-Vila et al. (2010).

614 Finally, we observe that models 2, 3 and 4 predict very similar peak values of $C_{C}$ at $t=$

615448 (Figure 5e-f), in spite of the very different structure exhibited by the reaction terms in the

616 three models. This is a remarkable result, also considering that previous studies identify the peak

617 concentration of $\mathrm{C}$ as a key indicator of incomplete mixing in the considered setting (e.g.,

618 Chiogna and Bellin, 2013; Sanchez-Vila et al., 2010). We emphasize that the profiles associated

619 with models 2 and 3 are obtained through calibration against data of $\bar{c}_{C}^{*}$ (see Figure 5f) while

620 model 4 leads to a very similar result directly embedding available information on pore-scale

621 geometry and velocity field, i.e. without the need for any adjustable parameter. The reaction 
622 product concentration profile rendered by model 4 shows a change in concavity in the vicinity of

623 the concentration peak for short times (see, e.g., Figure 5e, related to $x \approx 70$ ). This behavior is

624 likely due to the numerical discretization of the integro-differential terms of the model. This

625 observation is also supported by considering that this feature vanishes for long times (see Figure $6265 f)$.

We consider an irreversible homogeneous reaction taking place in a two-dimensional

629 disordered porous medium where pore-scale geometry and velocity are known and study the

630 reactive transport regime characterized by $\mathrm{Da} \square \mathrm{Pe} \square$ 1, corresponding to a fast reaction taking

631 place under advection dominated conditions. We simulate the reactive transport process at pore-

632 scale and we consider the pore-scale numerical results as calibration and validation data for 633 continuum-scale models.

634 We consider three effective models already presented in the literature which require 635 calibration against observed concentration profiles. Our results show that estimates of effective 636 reaction parameters describing incomplete mixing can be remarkably sensitive to the type of 637 concentrations one employs for model calibration, i.e., parameter estimates can be different 638 depending on whether the concentration of the reaction product or of one of the two reactants is 639 employed. This suggests that none of these models include a robust description of the way the 640 totality of pore-scale processes are transferred to continuum-scale formulations in the porous 641 medium we investigate. The estimated parameter values which model the effect of incomplete 642 mixing in the reaction term are characterized by large uncertainty when we employ the 643 concentration of the injected reactant for model calibration. This result suggests that information 
644 on concentration of the reaction product is required for a reliable (continuum-scale)

645 characterization of incomplete mixing in the considered setting.

646 We numerically solve a continuum-scale nonlocal model resulting from an upscaling of

647 the reactive transport setting considered via volume averaging. This model allows predicting the

648 longitudinal distribution of the reactants and of the reaction product without the need of any

649 adjustable parameter, solely requiring available information on pore-scale geometry and velocity.

650 This model is computationally intensive, as compared to the remaining three effective models we

651 analyze, but allows interpreting specific features observed from direct averaging of the pore-

652 scale numerical solution, such as the non-Fickian transport behavior of the invading reactant B

653 and the asymmetric shape of the reaction product concentration profile.

654 The considered two-dimensional porous system is characterized by a geometry which

655 includes large cavities where the reactant A, initially residing in the system, is trapped for long

656 times. All considered continuum-scale models fail to reproduce residual (normalized)

657 concentrations of $\mathrm{A}$ in the system below values of about 3-5\%. As a consequence, they are not

658 able to capture the details of the system behavior at large residence times associated with the

659 presence of minute values of trapped mass of solute A. It might be possible that the use of 660 double- or multi-continuum formulations of this reactive transport setting lead to improved 661 interpretation of this specific feature. This aspect will be investigated in future contributions.

\section{ACKNOWLEDGMENT}

The financial support of the MIUR Project PRIN 2010/2011 “Hydroelectric energy by

664 osmosis in coastal areas" is gratefully acknowledged. We acknowledge the contribution of 665 Sergey Chaynikov to the pore-scale numerical simulations. 
667 Alhashmi Z, Blunt BJ, Bijeljic B. Predictions of dynamic changes in reaction rates as a 668 consequence of incomplete mixing using pore-scale reactive transport modeling on images of 669 porous media. J Contam Hydrol 2015; doi: 10.1016/j.jconhyd.2015.06.004.

670 Battiato I, Tartakovsky DM. Applicability regimes for macroscopic models of reactive transport 671 in porous media. J Contam Hydrol 2011;120-1:18-26.

672 Bear J, Cheng A. Modeling groundwater flow and contaminant transport. Springer Science \& 673 Business Media; 2010.

674 Bekri S, Thovert J, Adler P. Dissolution of porous media. Chem Eng Sci 1995;50(17):2765-91.

675 Bijeljic B, Raeini A, Mostaghimi P, Blunt MJ. Predictions of non-Fickian solute transport in 676 different classes of porous media using direct simulation on pore-scale images. Phys Rev E $677 \quad 2013 ; 87(1): 013011$.

678 Carrera J, Neuman SP. Estimation of aquifer parameters under transient and steady state 679 conditions: 1. Maximum likelihood method incorporating prior information, Wat Resour Res. $680 \quad 1986,22(2): 199-210$.

681 Chastanet J, Wood BD. Mass transfer process in a two-region medium, Water Resour Res. $682 \quad 2008 ; 44$, W05413.

683 Chiogna G, Bellin A. Analytical solution for reactive solute transport considering incomplete 684 mixing within a reference elementary volume. Water Resour Res. 2013;49(5):2589-600.

685 Ciriello V, Edery Y, Guadagnini A, Berkowitz B. Multimodel framework for characterization of 686 transport in porous media. Water Resour Res. 2015;51(5):3384-3402.

687 Coelho D, Thovert J, Adler PM. Geometrical and transport properties of random packings of $688 \quad$ spheres and aspherical particles. Phys Rev E. 1997;55(2):1959-78. 
De Anna P, Jimenez-Martinez J, Tabuteau H, Turuban R, Le Borgne T, Derrien M, et al. Mixing and reaction kinetics in porous media: An experimental pore-scale quantification. Environ Sci Technol. 2013;48(1):508-16.

692 De Anna P, Dentz M, Tartakovsky A, Le Borgne T. The filamentary structure of mixing fronts and its control on reaction kinetics in porous media flows. Geophys Res Lett. 2014;41(13):4586-93.

Debenest G, Mourzenko VV, Thovert J-F. Smouldering in fixed beds of oil shale grains. A threedimensional microscale numerical model. Combust Theor Model. 2005;9: 113-35.

Ding D, Benson DA, Paster A, Bolster D. Modeling bimolecular reactions and transport in porous media via particle tracking. Adv Water Resour. 2013;53:56-65.

Edery Y, Dror I, Scher H, Berkowitz B. Anomalous reactive transport in porous media: Experiments and modeling. Phys Rev E. 2015;91(5):052130.

Edery Y, Guadagnini A, Scher H, Berkowitz B. Reactive transport in disordered media: Role of

Edery Y, Scher H, Berkowitz B. Modeling bimolecular reactions and transport in porous media.

Edery Y, Scher H, Berkowitz B. Particle tracking model of bimolecular reactive transport in fluctuations in interpretation of laboratory experiments. Adv Water Resour 2013;51:86-103. Geophys Res Lett. 2009;36(2) L02407.

Gramling CM, Harvey CF, Meigs LC. Reactive transport in porous media: A comparison of porous media. Water Resour Res. 2010;46(7):W07524.

model prediction with laboratory visualization. Environ Sci Technol. 2002;36(11):2508-14.

Guo J, Quintard M, Laouafa F. Dispersion in Porous Media with Heterogeneous Nonlinear Reactions. Transp Porous Med 2015; in press doi:10.1007/s11242-015-0535-4. 
711 Haggerty R, Harvey CF, von Schwerin CF, Meigs LC. What controls the apparent timescales in 712 aquifers and soils? A comparison of experimental results, Water Resour Res 2004;40:W01510.

714 Hansen SK, Scher H, Berkowitz B. First-principles derivation of reactive transport modeling 715 parameters for particle tracking and PDE approaches. Adv Water Resour. 2014;66:146-158.

716 Hecht F. New development in Freefem++, J. Numer. Math. 2012;20(3-4):251-266, 717 doi:10.1515/jnum-2012-0013.

718 Hochstetler DL, Kitanidis PK. The behavior of effective rate constants for bimolecular reactions 719 in an asymptotic transport regime. J Contam Hydrol. 2013;144(1):88-98.

720 Jose SC, Cirpka OA. Measurement of mixing-controlled reactive transport in homogeneous 721 porous media and its prediction from conservative tracer test data. Environ Sci Technol. 2004;38(7):2089-2096.

723 Kapoor V, Gelhar LW, Miralles-Wilhelm F. Bimolecular second-order reactions in spatially 724 varying flows: Segregation induced scale-dependent transformation rates. Water Resour Res $725 \quad 1997 ; 33(4): 527-36$.

726 Loyaux-Lawniczak S, Lehmann F, Ackerer P. Acid/base front propagation in saturated porous 727 media: 2D laboratory experiments and modeling. J Contam Hydrol. 2012;138-9:15-21.

728 Menke HP, Bijeljic B, Andrew MG, Blunt MJ. Dynamic three-dimensional pore-scale imaging 729 of reaction in a carbonate at reservoir conditions. Environ Sci Technol. 2015;49(7):4407-14.

730 Molins S, Trebotich D, Yang L, Ajo-Franklin JB, Ligocki TJ, Shen C, et al. Pore-scale controls 731 on calcite dissolution rates from flow-through laboratory and numerical experiments. 732 Environ Sci Technol. 2014;48(13):7453-60. 
733 Moyne, C. Two-equation model for a diffusive process in porous media using the volume 734 averaging method with an unsteady-state closure, Adv. Water Resour. 1997;20: 63-76.

735 Orgogozo L, Golfier F, Buès M, Quintard M. Upscaling of transport processes in porous media 736 with biofilms in non-equilibrium conditions, Adv. Water Resour. 2010;33: 585-600.

737 Porta GM, Chaynikov S, Thovert J, Riva M, Guadagnini A, Adler PM. Numerical investigation 738 of pore and continuum-scale formulations of bimolecular reactive transport in porous media. 739 Adv Water Resour. 2013;62:243-53.

740 Porta GM, Riva M, Guadagnini A. Upscaling solute transport in porous media in the presence of 741 an irreversible bimolecular reaction. Adv Water Resour. 2012;35:151-62.

742 Porta GM, Thovert J, Riva M, Guadagnini A, Adler P. Microscale simulation and numerical 743 upscaling of a reactive flow in a plane channel. Phys Rev E. 2012;86(3):036102.

744 Porta GM, Bijeljic B, Blunt MJ, Guadagnini A. Continuum-scale characterization of solute 745 transport based on pore-scale velocity distributions. Geophys Res Lett. 2015;42(18):75377467545.

747 Raje DS, Kapoor V. Experimental study of bimolecular reaction kinetics in porous media. $748 \quad$ Environ Sci Technol. 2000;34(7):1234-9.

749 Rolle M, Kitanidis PK. Effects of compound-specific dilution on transient transport and solute 750 breakthrough: A pore-scale analysis. Adv Water Resour. 2014; 71:186-99.

751 Rubio A, Zalts A, El Hasi C. Numerical solution of the advection-reaction-diffusion equation at 752 different scales. Environmental Modelling \& Software. 2008;23(1):90-5.

753 Sanchez-Vila X, Fernàndez-Garcia D, Guadagnini A. Interpretation of column experiments of 754 transport of solutes undergoing an irreversible bimolecular reaction using a continuum 755 approximation. Water Resour Res. 2010;46(12). 
756 Valdés-Parada FJ, Aguilar-Madera CG, Álvarez-Ramírez J. On diffusion, dispersion and reaction 757 in porous media. Chem Eng Sci 2011;66: 2177-90. doi:10.1016/j.ces.2011.02.016.

758 Whitaker S. The method of volume averaging. Springer Science \& Business Media; 1998.

759 Willingham T, Zhang C, Werth CJ, Valocchi AJ, Oostrom M, Wietsma TW. Using dispersivity 760 values to quantify the effects of pore-scale flow focusing on enhanced reaction along a 761 transverse mixing zone. Adv Water Resour. 2010;33(4):525-35.

762 


\begin{tabular}{|c|c|c|c|c|c|c|c|}
\hline & & Model 1 & \multicolumn{3}{|c|}{ Model 2} & \multicolumn{2}{|c|}{ Model 3} \\
\hline Data & & $D$ & $D$ & $\hat{\beta}_{0}$ & $M$ & $D$ & $\lambda$ \\
\hline \multirow{3}{*}{$\bar{c}_{B}^{*}$} & Best estimate & 698 & 678 & 0.16 & 0.08 & 510 & 87 \\
\hline & $\sigma_{p}$ & 50 & 45 & 2.23 & 0.25 & 46 & 149 \\
\hline & $J_{B \min }$ & 0.0102 & \multicolumn{3}{|c|}{0.0093} & \multicolumn{2}{|c|}{0.0194} \\
\hline \multirow{3}{*}{$\bar{c}_{C}^{*}$} & Best estimate & 388 & 423 & 0.04 & 0.76 & 415 & 2.08 \\
\hline & $\sigma_{p}$ & 36 & 56 & 1.019 & 0.24 & 34 & 1.36 \\
\hline & $J_{\text {Cmin }}$ & 0.0102 & \multicolumn{3}{|c|}{0.0071} & \multicolumn{2}{|c|}{0.0071} \\
\hline
\end{tabular}

763 Table 1: Results of effective model calibration: best estimate of model parameters, related

764 standard deviation $\sigma_{p}$, as evaluated through the diagonal entries of $\mathbf{Q}$ (32), and associated

765 minimum values of metrics (30)-(31). 
FIGURES

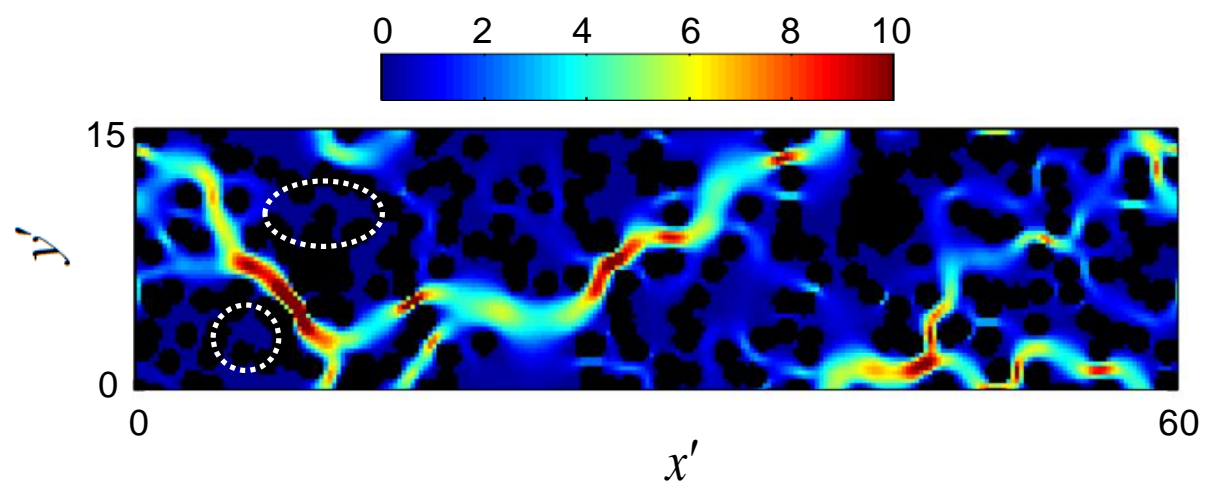

771 Figure 1. Distribution of velocity $|\mathbf{u}|=|\hat{\mathbf{u}}| / \hat{U}$ within a unit cell $\Omega^{\prime}$. Areas enclosed by the white dotted circles correspond to cavities characterized by low velocity. 

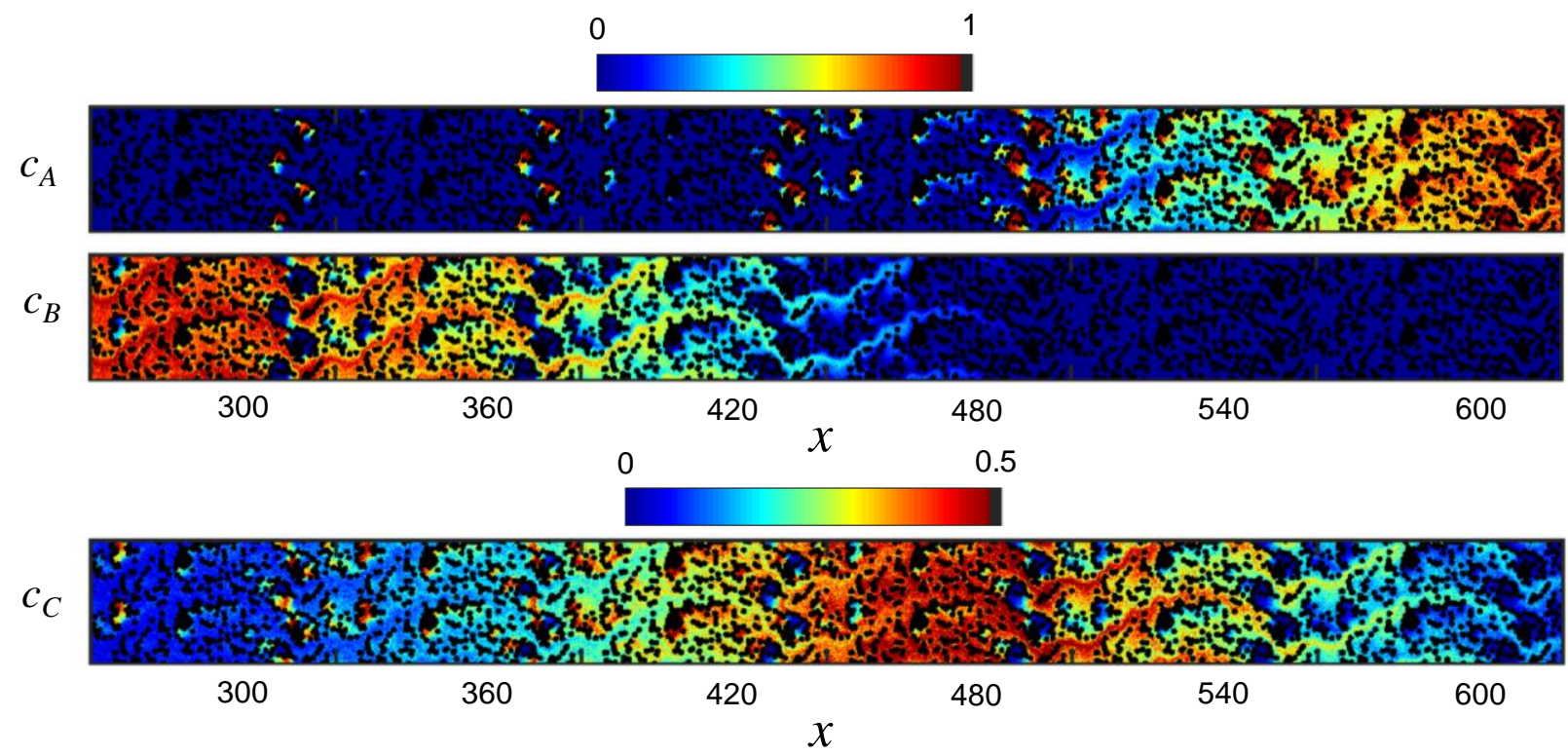

774

775 Figure 2. Snapshot of the pore-scale concentration fields $c_{A}, c_{B}$ and $c_{C}$ at time $t=448$.

776 


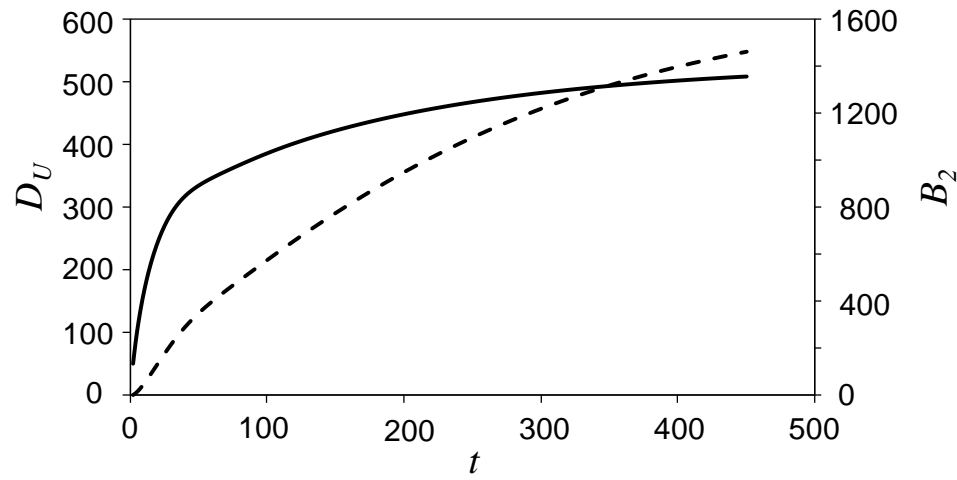

778 Figure 3. Temporal evolution of the effective dispersion coefficient $D_{U}(16)$ (continuous curve,

779 left vertical axis) and of $B_{2}$ (26) (dashed curve, right vertical axis).

780 

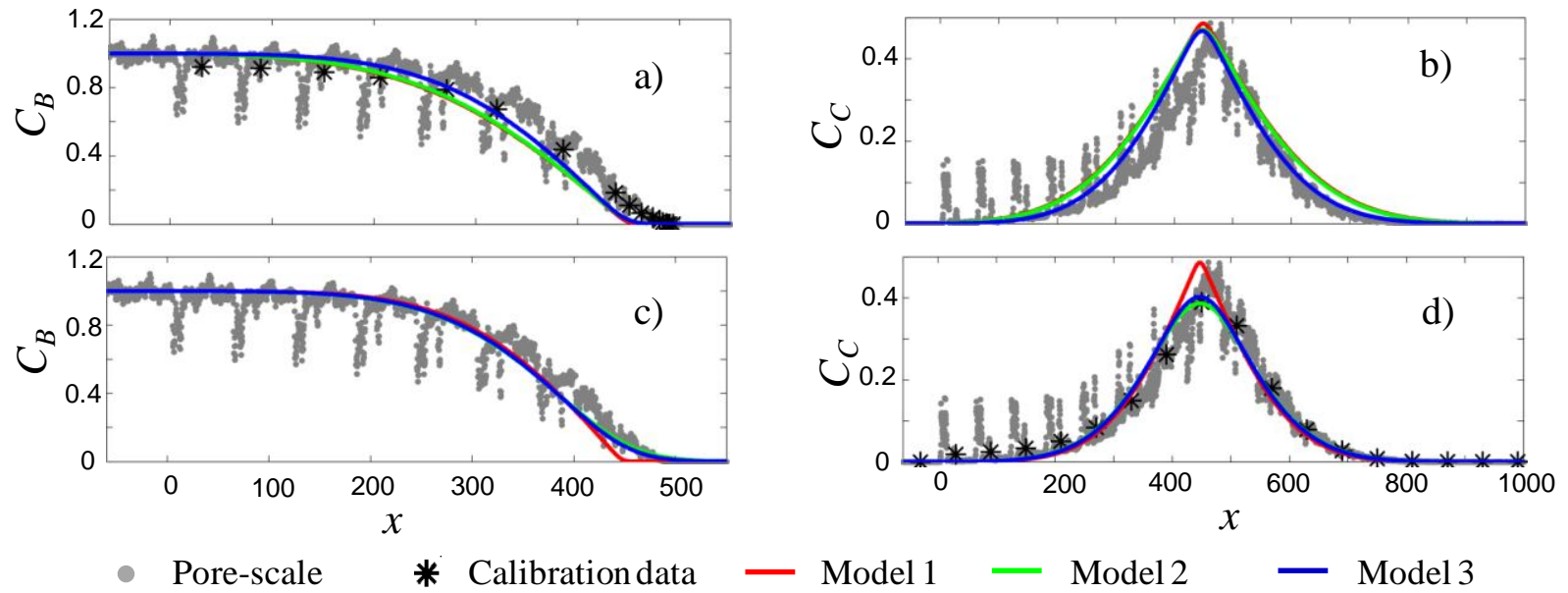

Model 1

Model 2

- Model 3

782 Figure 4. Model calibration results: concentration profiles resulting from model calibration

783 (continuous curves) against data sets $\bar{c}_{B i}^{*}$ (a-b) and $\bar{c}_{C i}^{*}$ (c-d); calibration data $\bar{c}_{B i}^{*}$ (black symbols

784 in a) and $\bar{c}_{C i}^{*}$ (black symbols in d) and section-averaged concentrations resulting from pore-scale

785 simulations (grey points) 

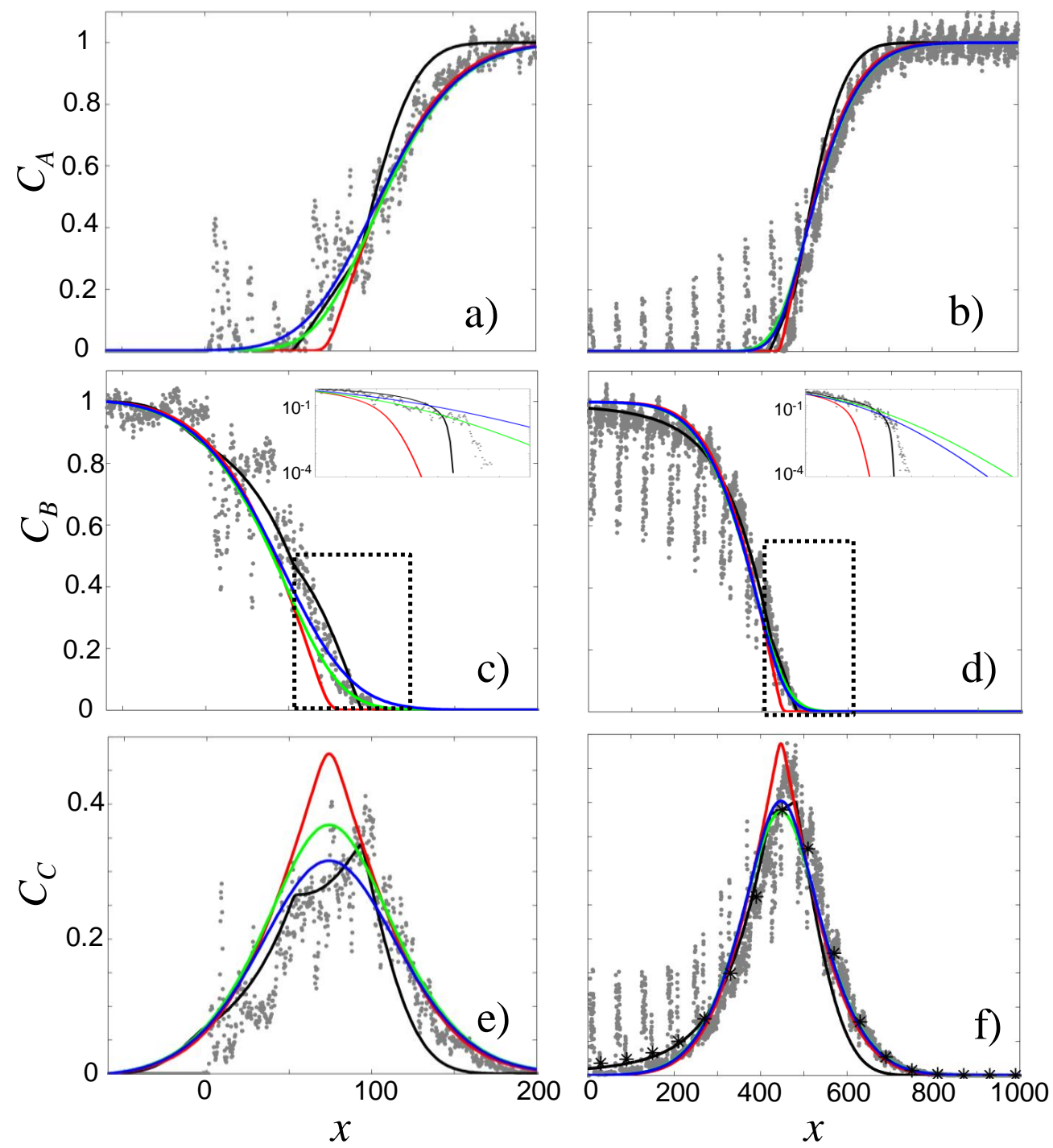

- Pore-scale

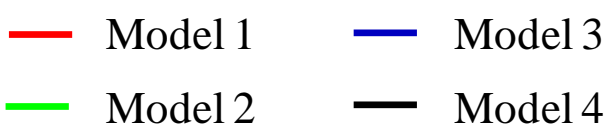

788 Figure 5. Comparison between section-averaged pore-scale concentrations and continuum-scale 789 model results obtained for reactants $\mathrm{A}(\mathrm{a}-\mathrm{b}), \mathrm{B}(\mathrm{c}-\mathrm{d})$ and reaction product $\mathrm{C}$ (e-f), for 790 dimensionless times $t=47$ (left column: a,c,e) and $t=448$ (right column: b,d,f). Results of 791 models 1-3 are obtained through model calibration on $\bar{c}_{C i}^{*}$ data (black symbols in $\mathrm{f}$ ). Insets in c-d 792 display a zoom (in semi-logarithmic scale) of the regions identified by the dot-dashed boxes. 

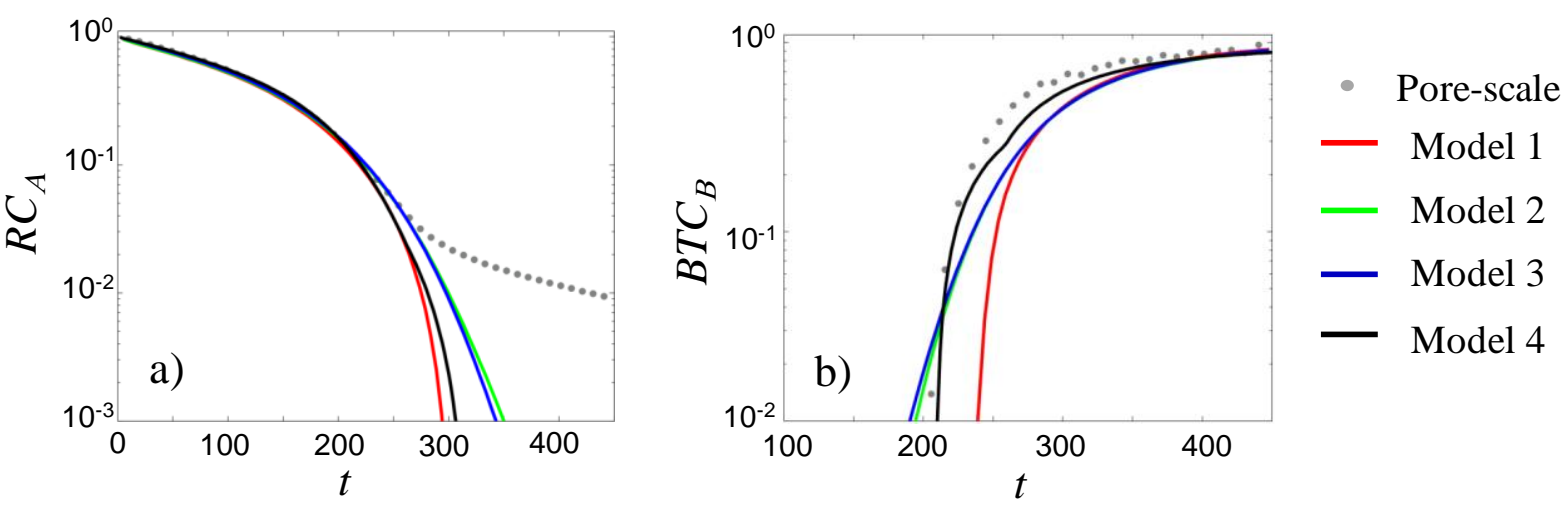

795

796 Figure 6. Temporal evolution of (a) flux-weighted concentration of reactant B $\left(B T C_{B}\right)$ at $X_{M}=$ 797300 and (b) residual concentration of species A $\left(R C_{A}\right)$ evaluated with $X_{M}=300$. 

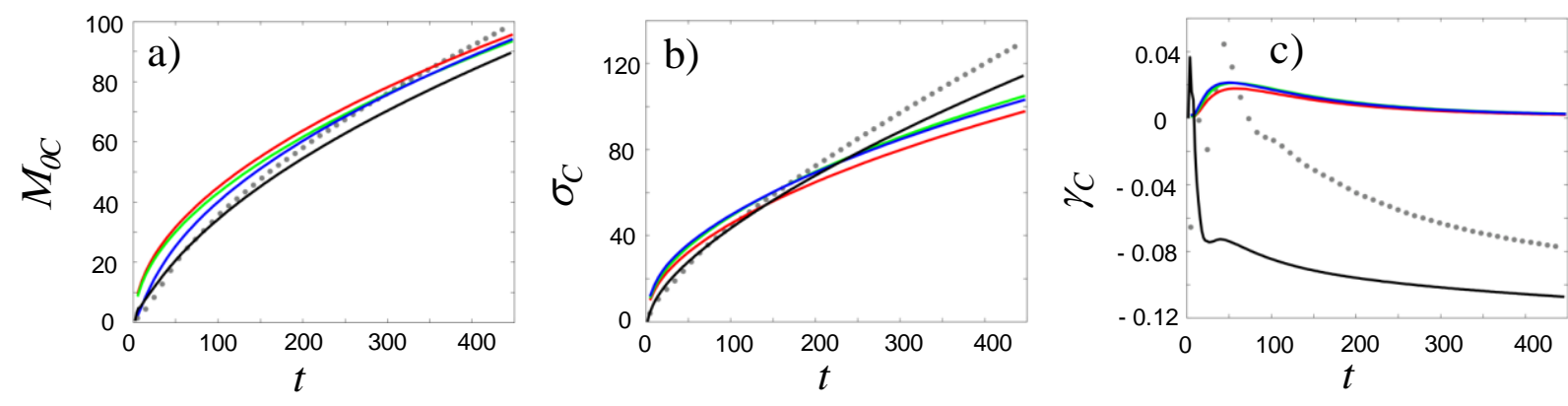

800

- Pore-scale $\quad$ Model 1

Model 2

Model 3

- Model 4

801 Figure 7. Temporal evolution of (a) the total mass, (b) spreading and (c) skewness associated

802 with the spatial distribution of the reaction product $\mathrm{C}$ as given by the considered continuum-scale

803 models (lines) and pore-scale simulation (symbols). 